\title{
A review on "Experimental investigation of a low-temperature organic Rankine cycle (ORC) engine under variable heat input operating at both subcritical and supercritical conditions"
}

\author{
Aishwarya A. Gavai ${ }^{* *}$ \\ ${ }^{\dagger}$ Mechanical Department, MITCOE, Pune. India \\ Accepted 02 March 2016, Available online 15 March 2016, Special Issue-4 (March 2016)
}

\begin{abstract}
The research paper citied, involves detailed experimental investigation of an organic Rankine cycle (ORC), which is designed to operate at supercritical conditions. The tests are conducted at the laboratory and the heat source is a controllable electric heater, which can keep the hot water temperature constant, by switching on/off its electrical resistances. The expansion machine is a modified scroll compressor with major conversions, in order to be able to operate with safety at high pressure (or even supercritical at some conditions). The ORC engine is equipped with a dedicated heat exchanger of helical coil design, suitable for such applications. The speeds of the expander and ORC pump are regulated with frequency inverters, in order to control the cycle top pressure and heat input. The performance of all components is evaluated, while special attention is given on the supercritical heat exchanger and the scroll expander.
\end{abstract}

Keywords: Organic Rankine cycle, engine, subcritical, supercritical

\section{Introduction}

The organic Rankine cycle (ORC) technology is suitable for heat recovery applications for low-temperature of even lower than $100^{\circ} \mathrm{C}$

The main advantage at this temperature range is the simple and low-cost heat source circuit, since even pure water can be used with low-pressure piping, while the use of glycol or thermal oil is avoided, as well as a simple ORC configuration with a single expansion machine and no internal heat exchangers.

There are various studies focusing on supercritical ORC at theoretical level. Here, an experimental study is implemented, testing a smallscale supercritical ORC with a net capacity of $3 \mathrm{~kW}$ for variable heat input and for hot water temperature equal to $95{ }^{\circ} \mathrm{C}$. The measured data are presented and discussed, focusing on the expansion machine and the whole engine operation, as well as its capability to reach supercritical operation at some conditions.

\section{Performance test}

The goal is to examine and evaluate the ORC engine performance at these conditions, while supercritical operation is also attempted, in order to identify if and how much the efficiency can be increased at such

${ }^{*}$ Corresponding author: Aishwarya A. Gavai unexplored conditions, especially for small scale engines. The HTF temperature is set constant to $95^{\circ} \mathrm{C}$, while the heat input is varied.

\section{Subtopics being addressed}

The installed ORC engine

A small-scale ORC engine has been designed and constructed. The engine cooling is accomplished with a cooling water circuit, using a conventional shell and tube heat exchanger. The ORC engine has been then installed at the laboratory for performance tests under controlled conditions.

\subsection{Subcritical operation}

Most of the tests have been conducted at subcritical operation. The temperature of the organic fluid at the evaporator outlet was at all times over its critical temperature (equal to $72.1{ }^{\circ} \mathrm{C}$ ), while the top cycle pressure was below the critical pressure (equal to 37.29 bar). The operating conditions that have been examined concern the variation of the ORC pump and expander speeds, which in turn affect the cycle and fluid properties especially at the pump, vapor generator and expander. 


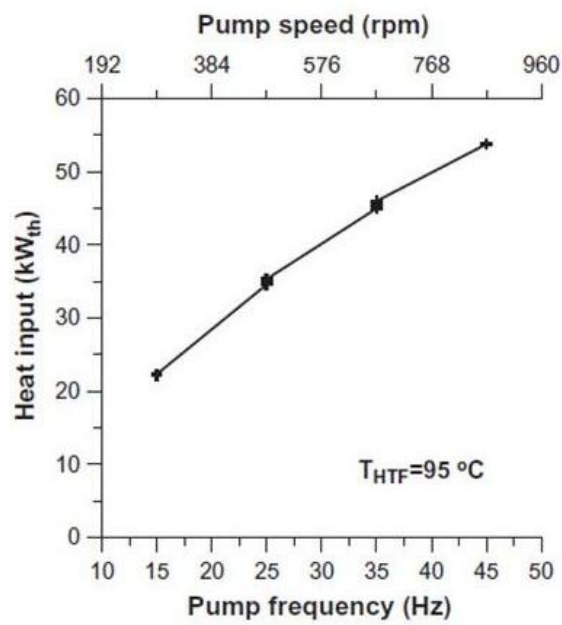

Fig. 1: Pump frequency v/s Heat input v/s Pump speed

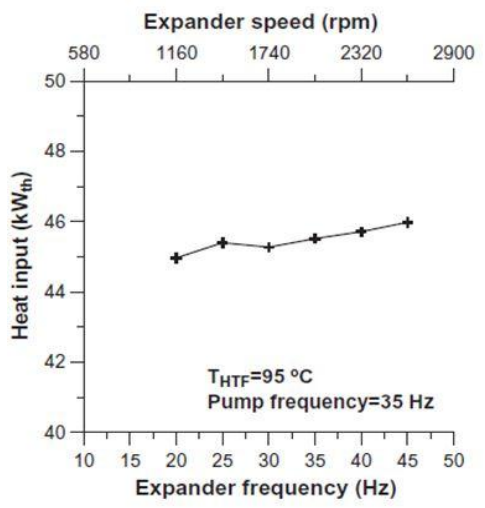

Fig. 2: Expander frequency v/s Heat input v/s Expander speed

\section{Supercritical operation}

Supercritical pressure could be reached only for very high pump speed and low expander speed. Low value was expected, since the low expander speed leads to a low electric efficiency of the induction generator. Nevertheless, this efficiency value is much higher than all the other recorded in the same engine and for such low expander speed (usually in the range of 1.5-2\%), and provides a first positive indication for the supercritical cycle, even if few such operating conditions have been available. The thermal efficiency is also depicted in where it is shown that the maximum efficiency reached is almost $6 \%$ for subcritical operation, while for supercritical operation was around $4.4 \%$.

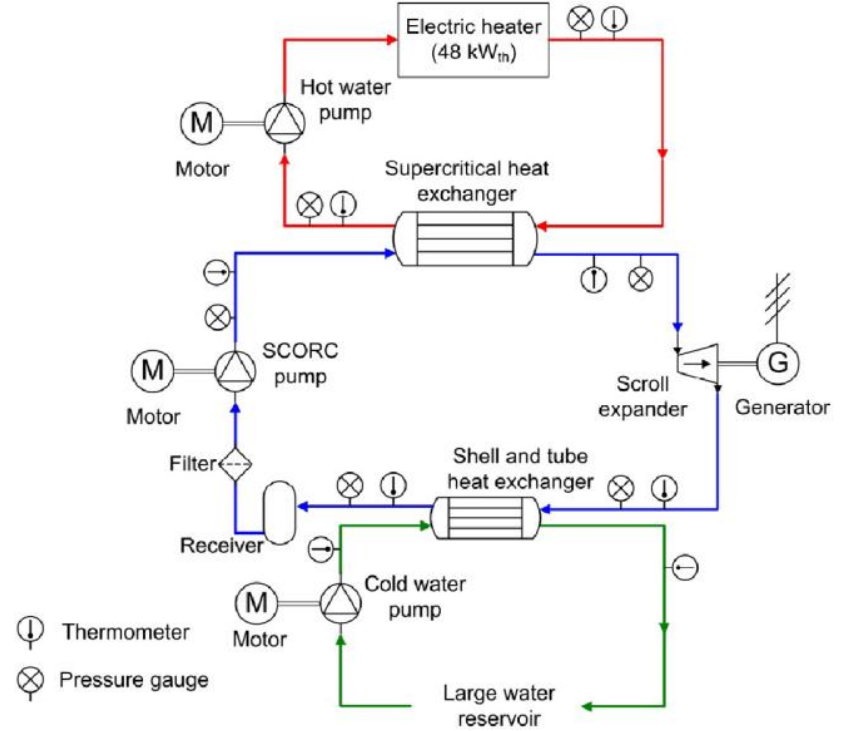

Fig. 3: Expander frequency v/s Heat input v/s Expander speed

\section{Conclusion}

In the present study real test data are presented, and the advantages and potential of such technology could be identified. Also, some first proof is provided, whether a supercritical ORC can be indeed more efficient than a subcritical cycle, and if the theoretical results can be verified.

\section{References}

George Kosmadakis , Dimitris Manolakos, George Papadakis Department of Natural Resources and Agricultural Engineering, Agricultural University of Athens, Iera Odos Street 75, Athens 11855, Greece

George Kosmadakis, Dimitris Manolakos, George Papadakis, (Oct 2015)Experimental investigation of a low-temperature organic Rankine cycle (ORC) engine under variable heat input operating at bothsubcritical and supercritical conditions, Applied Thermal Engineering 92 (2016) 1-7, Elsevier

Wasun Darawun, Roongrojana Songprakorp, Veerapol Monyakula, Sirichai Thepa, (2015), International Conference on Alternative Energy in Developing Countries and Emerging Economies, Energy Procedia 